\title{
Separable Coordinates for Four-Dimensional Riemannian Spaces
}

C. P. Boyer

IIMAS, Universidad Nacional Autonoma de Mexico, Mexico 20, D.F., Mexico

E. G. Kalnins

Mathematics Department, University of Waikato, Hamilton, New Zealand

Willard Miller, Jr.

School of Mathematics, University of Minnesota, Minneapolis, Minnesota 55455, USA

Abstract. We present a complete list of all separable coordinate systems for the equations $\sum_{i, j=1}^{4} g^{-1 / 2} \partial_{i}\left(g^{1 / 2} g^{i j} \partial_{j} \Phi\right)=E \Phi$ and $\sum_{i, j=1}^{4} g^{i j} \partial_{i} W \partial_{j} W=E$ with special emphasis on nonorthogonal coordinates. Applications to general relativity theory are indicated.

\section{Introduction}

We study the problem of separation of variables for the equations
a) $\Delta_{4} \Phi=\sum_{i, j=1}^{4} \frac{1}{\sqrt{g}} \partial_{i}\left(\sqrt{g} g^{i j} \partial_{j} \Phi\right)=E \Phi$
b) $\sum_{i, j=1}^{4} g^{i j} \partial_{i} W \partial_{j} W=E$.

Here, $d s^{2}=\sum g_{i j} d x^{i} d x^{j}$ is a complex Riemannian metric, $g=\operatorname{det}\left(g_{i j}\right), \sum_{j} g^{i j} g_{j k}=\delta_{k}^{i}$, $g_{i j}=g_{j i}$, and $E$ is a nonzero complex constant. (Furthermore, we have adopted the notation $\partial_{i} W=W_{i}=\frac{\partial W}{\partial x^{i}}$. $)$ Thus (1.1a) is the Helmholtz equation on a four dimensional complex Riemannian space and (1.1b) is the associated HamiltonJacobi (HJ) equation.

In this paper we classify all metrics and coordinate systems for which Equations (1.1) admit solutions via separation of variables. For (1.1a) the separation is in terms of a product whereas for (1.1b) it is in terms of a sum

$$
\Phi(\mathbf{x})=\prod_{i=1} \Phi^{(i)}\left(x^{i}\right), \quad W(\mathbf{x})=\sum_{i=1}^{4} W^{(i)}\left(x^{i}\right) .
$$


By applying appropriate reality conditions on the metric tensor we can use our results to obtain the separable and partially separable systems for corresponding real equations such as the Euclidean space Helmholtz equation, the Minkowski space Klein-Gordon equation [1] and various equations of general relativity theory. Indeed the interest in solving relativistic equations via separation of variables methods, see e.g. [2-5], is motivation for our work. In a forthcoming paper we shall make these applications explicit by classifying all separable systems for (1.1a) and (1.1b) in Ricci-flat spaces.

There is a deep relationship between the symmetry groups of Equations (1.1), the coordinate systems in which these equations admit solutions via separation of variables and the properties of the separated (special function) solutions so obtained. See [6] for an examination of this correspondence in the case of some of the most common partial differential equations of mathematical physics. We mention in particular that the myriad addition theorems, generating functions and expansion formulas for the special functions of mathematical physics can be derived systematically in terms of this relationship. Thus the method of separation of variables assumes an importance far beyond the fact that it permits the construction of explicit solutions for partial differential equations.

The classical Stäckel method for separating variables in Equations (1.1) is well known, [7], [8]. We briefly review the main ideas for the HJ equation (1.1b). Let $\left\{x^{i}\right\}$ be a prospective separable system for the HJ equation with separated ordinary differential equations

$$
\Phi_{i} \equiv f_{i}\left(x^{i}\right)\left(W^{\prime(i)}\right)^{2}+g_{i}\left(x^{i}\right) W^{\prime(i)}+h_{i}\left(x^{i}\right)+\sum_{j=1}^{4} c_{j} S_{i j}\left(x^{i}\right)=0 \quad i=1, \ldots, 4 .
$$

Here $c_{1}=-E$ and $c_{2}, c_{3}, c_{4}$ are the separation constants. It is assumed that the Stäckel determinant $S=\operatorname{det}\left(S_{i j}\right)$ is nonzero. Furthermore, if $f_{i} \neq 0$ we can require that $f_{i} \equiv 1$. To relate (1.3) with (1.1b) one looks for functions $\Theta_{i}\left(x^{1}, \ldots, x^{4}\right)$ such that

$$
\sum_{i=1}^{4} \Theta_{i} \Phi_{i} \equiv \sum_{i, j=1}^{4} g^{i j} \partial_{i} W \partial_{j} W-E
$$

identically in the separation constants $E, c_{2}, c_{3}, c_{4}$. Stäckel [8] showed that the only solution is $f_{i} \equiv 1, g_{i} \equiv h_{i} \equiv 0, \Theta_{i}=M_{j 1} / S$ where $M_{j 1}$ is the (j1) cofactor of $S$. In particular the metric must be orthogonal.

The classification procedure utilized here is more general than that of Stäckel and is based on a method introduced in [9] for three-dimensional Riemannian manifolds. Here the separable systems are classified in terms of the number of ignorable coordinates they contain. (A variable $x^{i}$ in a separable system is termed ignorable if $\partial_{i} g_{j k}=0$ for $1 \leqq j, k \leqq 4$, i.e., the metric tensor is independent of $x^{i}$. Otherwise the variable $x^{i}$ is essential. If the separated ordinary differential equation in the essential variable $x^{i}$ is first order in $W^{(i)}$ then $x^{i}$ is of type 1 , if second order then $x^{i}$ is of type 2 .

If $x^{4}$ is ignorable then the $\mathrm{HJ}$ equation admits solutions of the form $W=$ 
$\tilde{W}\left(x^{1}, x^{2}, x^{3}\right)+c_{4} x^{4}$ where $c_{4}$ is a constant, and $(1.1 \mathrm{~b})$ reduces to

$$
\sum_{i, j=1}^{3} g^{i j} \partial_{i} W \partial_{j} W+2 c_{4} \sum_{j=1}^{3} g^{4 j} \partial_{j} W+c_{4}^{2} g^{44}=E,
$$

an equation in three variables. If all remaining variables are required to be essential, we can then apply the Stäckel method to (1.5) and find an equivalent system of three ordinary differential Equations (1.3) where now $i=1,2,3, j$ is summed from 1 to 3 and $f_{i}$ need not be nonzero. If, however, two, three or four variables are ignorable we can first split off these variables, introducing a separation constant $c_{k}$ for each ignorable variable, and apply the Stäckel method to the reduced equation in the remaining essential variables.

The orthogonal separable systems we obtain are exactly those which one would find by employing the classical Stäckel method. However, for systems with at least one ignorable variable we find truly nonorthogonal coordinates which do not seem to appear in the literature. Our lists are also simpler and more explicit than those given heretofore. This is primarily because, as pointed out in [9], one can identify all coordinate systems which lead to the same families of separable solutions for Equations (1.1). (For example, if $\left\{x^{i}\right\}$ is a separable system with $x^{4}$ ignorable and $x^{1}, x^{2}, x^{3}$ essential then it is easy to see that $\left\{X^{i}\right\}$ with $X^{j}=x^{j}, j=1,2,3, X^{4}=x^{4}+a_{1}\left(x^{1}\right)+a_{2}\left(x^{2}\right)+a_{3}\left(x^{3}\right), a_{l}$ arbitrary, is also a separable system with $X^{4}$ ignorable and $X^{1}, X^{2}, X^{3}$ essential. Furthermore, the two systems have the same separable solutions. We regard all such systems as equivalent and merely give one representative from each equivalence class in our lists. The functions $a_{l}$ are chosen such that this representative is as simple as possible. In particular, if possible we choose the $a_{l}$ such that the resulting metric is orthogonal. If this cannot be done we say that the separable system is truly nonorthogonal. All other separable systems are equivalent to orthogonal separable systems. Similar remarks hold for two, three and four ignorable coordinates.)

In this paper we list all possible ways variables can locally separate on a four-dimensional Riemannian manifold. A related problem not solved here is: Given a particular manifold $M$ list all separable systems for $M$. To solve this problem we must determine which of the metrics listed here can be interpreted as a metric for $M$. In particular, for flat space we must require that the curvature tensor corresponding to each separable metric vanish identically. For orthogonal separable systems we have solved this problem in flat spaces $E_{4},[1]$ and in the space of constant curvature $S_{4},[10]$. For nonorthogonal separable systems in $E_{4}$ and $S_{4}$ the solutions will be given in forthcoming articles.

The most notable paper concerning separation of variables for Equations (1.1) is undoubtedly that by Eisenhart [11]. It follows easily from Stäckel's original paper [8] that any orthogonal separable system for (1.1a) also separates (1.1b). Robertson [12] found a necessary and sufficient condition that a separable system for (1.1b) also separate (1.1a). Eisenhart's great contribution was to show that the Robertson condition amounted to the requirement $R_{i j}=0$ for $i \neq j$ where $R_{k l}$ is the Ricci tensor of the Riemann manifold. It follows immediately from this result that for all Einstein spaces (in particular for flat space and spaces of 
constant curvature) the Helmholtz and $\mathrm{HJ}$ equations separate in exactly the same orthogonal coordinate systems. Furthermore, this result makes feasible the computation of all separable orthogonal systems for a given Riemann manifold provided the curvature tensor of the manifold can be characterized in a reasonably simple manner.

Among recent contributors, Havas has influenced us the most. In his papers [13] and [14] he gave a useful summary of the classical work relating Equations (1.1), listed both orthogonal and nonorthogonal separable (and partially separable) systems for these equations in $n$ variables and emphasized the relevance of the condition $R_{i j}=0, i \neq j$, even for the Helmholtz separability of nonorthogonal systems. Our work differs from his primarily in the explicit nature of our results and in the new nonorthogonal systems we find. Most of the nonorthogonal systems obtained by Havas and earlier workers appear to be equivalent to orthogonal separable systems in the sense discussed in [9]. In this paper we show that the condition $R_{i j}=0, i \neq j$, for all non-ignorable variables $x^{i}, x^{j}$ is necessary and sufficient for Helmholtz separability of even nonorthogonal $\mathrm{HJ}$ separable systems with two exceptions, the nonorthogonal E1 and E2 systems. These special systems, particularly E1, are important counterexamples for several conjectures concerning separable coordinates.

There is another series of papers [3-5], based on some results of Woodhouse, concerning separability of Equations (1.1) and explicitly pointing out the relevance of variable separation to the symmetry properties of (1.1) and to modern relativity theory. However, the authors of these papers adopt a very special definition of separation and partial separation of variables which rules out many of the classical separable systems. They consider only the type of variable separation in which one variable can be explicitly separated from the remaining variables in (1.1). This definition omits such well known separable systems as the Lame ellipsoidal coordinates and paraboloidal coordinates in flat space for which all variables must be separated simultaneously (i.e., the full Stäckel matrix machinery must be used [11]). The variable separation treated in these papers corresponds to our types I and J.

As a first step in the application of our results to relativity theory we establish in this paper that a Ricci-flat space admitting a separable coordinate system must also admit a Killing vector. Furthermore a separable system for a non-flat Ricciflat space must contain an ignorable coordinate.

For corresponding treatments of Equations (1.1) in three and four variables with $E=0$ see [15-17].

\section{Equations with Ignorable Coordinates}

We now enumerate the possible separable coordinate systems for the $\mathrm{HJ}$ and Helmholtz equations which contain at least one ignorable variable.

\section{A. All Variables Ignorable}

By applying a linear transformation $x^{i}=a_{j}^{i} \bar{x}^{j}$ to the ignorable coordinates $\left\{\bar{x}^{j}\right\}$ 
we can obtain an equivalent set of ignorable coordinates $\left\{x^{i}\right\}$ for which $g_{i j}=\delta_{i j}$. The $\mathrm{HJ}$ equation becomes

$$
\text { [A] } \quad W_{1}^{2}+W_{2}^{2}+W_{3}^{2}+W_{4}^{2}=E,
$$

and the corresponding Helmholtz equation is also separable in these flat space variables.

\section{B. Three Ignorable Variables}

If $x^{1}$ is the essential variable then $g_{i j}=G_{i j}\left(x^{1}\right)$. By re-defining the ignorable variables $\left\{\bar{x}^{j}\right\}$ according to $d x^{i}=d \bar{x}^{i}+h_{i}\left(x^{1}\right) d x^{1}$ where $g_{i j}=-\sum_{k=2}^{4} g_{j k} h_{k}, j=$ 2, 3,4 we can obtain the $\mathrm{HJ}$ equation

$$
\text { [B] } \quad W_{1}^{2}+\sum_{i, j=2}^{4} g^{i j}\left(x^{1}\right) W_{i} W_{j}=E .
$$

The corresponding Helmholtz equation also separates in these coordinates.

\section{Two Ignorable Variables with Two Essential Variables of Type 2}

We will treat this case in some detail to indicate our methods of derivation. If the essential variables are $x^{1}, x^{2}$ then for separation of the $\mathrm{HJ}$ equation the contravariant metric must have the form

$$
\begin{aligned}
& g^{11}=g^{22}=Q, g^{13}=Q a\left(x^{1}\right), g^{23}=Q b\left(x^{2}\right) \\
& g^{14}=Q c\left(x^{1}\right), g^{24}=Q d\left(x^{2}\right), g^{33}=Q\left[e_{1}\left(x^{1}\right)+e_{2}\left(x^{2}\right)\right] \\
& g^{44}=Q\left[f_{1}\left(x^{1}\right)+f_{2}\left(x^{2}\right)\right], g^{34}=Q\left[h_{1}\left(x^{1}\right)+h_{2}\left(x^{2}\right)\right], g^{12}=0
\end{aligned}
$$

where $Q=1 /\left[K_{1}\left(x^{1}\right)-K_{2}\left(x^{2}\right)\right]$. By defining equivalent ignorable coordinates $x^{3}, x^{4}$ to the original coordinates $\bar{x}^{3}, \bar{x}^{4}$ by

$$
d x^{3}=d \bar{x}^{3}-a d x^{1}-b d x^{2}, d x^{4}=d \bar{x}^{4}-c d x^{1}-d d x^{2}
$$

we can assume $a=b=c=d=0$ in (2.3). Thus the $\mathrm{HJ}$ equation becomes

$$
\text { [C] } \begin{gathered}
\frac{1}{K_{1}-K_{2}}\left[W_{1}^{2}+W_{2}^{2}+\left(e_{1}+e_{2}\right) W_{3}^{2^{*}}+2\left(h_{1}+h_{2}\right) W_{3} W_{4}\right. \\
\left.+\left(f_{1}+f_{2}\right) W_{4}^{2}\right]=E .
\end{gathered}
$$

The corresponding Helmholtz equation separates if and only if

$$
\partial_{12} \ln \left[\frac{\left(K_{1}-K_{2}\right)^{2}}{\left(e_{1}+e_{2}\right)\left(f_{1}+f_{2}\right)-\left(h_{1}+h_{2}\right)^{2}}\right]=0
$$

and this condition is equivalent to $R_{12}=0$ where $R_{i j}$ is the Ricci tensor. 
D. Two Ignorable Variables with One Essential Variable of Each Type

There are two cases to distinguish, the first being

$$
\begin{gathered}
\text { [D1] } \frac{1}{K_{1}-K_{2}}\left[W_{1}^{2}+2 a_{2} W_{2} W_{3}+2 b_{2} W_{2} W_{4}+d_{1} W_{3}^{2}\right. \\
\left.+2\left(f_{1}+f_{2}\right) W_{3} W_{4}+e_{1} W_{4}^{2}\right]=E,
\end{gathered}
$$

where the subscript $i$ on $a, b, d, e, f, K$ denotes the essential variable $x^{i}$ on which the function depends. Here $a_{2} b_{2} \neq 0$. The necessary and sufficient condition for separation of the corresponding Helmholtz equation is

$$
\partial_{12} \ln \left[\frac{\left(K_{1}-K_{2}\right)^{2}}{2 a_{2} b_{2}\left(f_{1}+f_{2}\right)-a_{2}^{2} e_{1}-b_{2}^{2} d_{1}}\right]=0,
$$

which is equivalent to $R_{12}=0$.

The second case corresponds to

$$
\text { [D2] } \begin{array}{r}
\frac{1}{K_{1}-K_{2}}\left[W_{1}^{2}+2 W_{2} W_{4}+\left(d_{1}+d_{2}\right) W_{3}^{2}\right. \\
\left.+2 f_{1} W_{3} W_{4}+e_{1} W_{4}^{2}\right]=E .
\end{array}
$$

The necessary and sufficient condition for separation of the Helmholtz equation is

$$
\partial_{12} \ln \left[\frac{\left(K_{1}-K_{2}\right)^{2}}{d_{1}+d_{2}}\right]=0
$$

and this is equivalent to $R_{12}=0$.

\section{E. Two Ignorable Variables with Two Essential Variables of Type 1}

Here there are three cases to distinguish. The first possibility is

[E1]

$$
\begin{gathered}
\frac{1}{K_{1}-K_{2}}\left[2 a_{1} W_{1} W_{3}+2 W_{1} W_{4}+2 a_{2} W_{2} W_{3}+2 W_{2} W_{4}\right. \\
\left.+\left(c_{1}+c_{2}\right) W_{3}^{2}\right]=E, a_{1} a_{2} \neq 0
\end{gathered}
$$

Necessary and sufficient conditions for separation of the Helmholtz equation are

$$
\begin{aligned}
& \partial_{12}\left(a_{1} \partial_{1} \psi+a_{2} \partial_{2} \psi\right)=\partial_{12}\left(\partial_{1} \psi+\partial_{2} \psi\right)=0, \\
& \psi=\ln \left(\frac{K_{1}-K_{2}}{a_{1}-a_{2}}\right) .
\end{aligned}
$$

In this case the condition $R_{12}=0$ where

$$
R_{12}=\partial_{12} \psi-\frac{1}{2} \partial_{1} \psi \partial_{2} \psi-\frac{1}{2} \frac{\left(a_{1}^{\prime}-a_{2}^{\prime}\right)}{a_{1}-a_{2}}\left(\partial_{1} \psi+\partial_{2} \psi\right)-\frac{1}{2} \frac{\left(a_{1}^{\prime \prime}-a_{2}^{\prime \prime}\right)}{a_{1}-a_{2}}
$$

neither implies nor is a consequence of (2.12). To show this we first find all solutions of Equations (2.12). The general solution of the second equation is

$$
\psi=f\left(x^{1}-x^{2}\right)+g\left(x^{1}\right)+h\left(x^{2}\right)
$$


and substituting this result into the first equation we obtain

Lemma 1. A coordinate system of type $\mathrm{E} 1$ permits separation of the Helmholtz equation if and only if it corresponds to one of the following three types:

i) $a_{1}=\cosh x^{1}, a_{2}=\cosh x^{2}, \psi=-\ln \left(e^{x^{2}-x^{1}}-1\right)$

ii) $a_{1}=e^{x^{1}}, a_{2}=e^{x^{2}}, K_{2}=0$

iii) $\partial_{12} \psi=0$.

It is easy to check that $R_{12} \neq 0$ for the systems of types i) and ii). Furthermore the system

$$
a_{1}=(3+\sqrt{5}) x^{1}, a_{2}=2 x^{2}, K_{1}=1, K_{2}=0
$$

satisfies $R_{12}=0$ but not Equations (2.12).

We briefly investigate the possible E1 systems which can occur in flat space. The only nonvanishing elements of the curvature tensor for an E1 system are $R_{2114}, R_{1223}, R_{2113}$ and $R_{1212}$. A direct computation shows that if the first three of these elements are required to be zero then the system satisfies $\partial_{12} \psi=0$, hence permits separation of the Helmholtz equation.

Lemma 2. A type E1 system in flat space permits separation of the flat space Helmholtz equation.

The second possibility for type E systems is

$$
\frac{1}{K_{1}-K_{2}}\left[2 W_{1} W_{4}+2 W_{2} W_{3}+2 b_{2} W_{2} W_{4}+\left(c_{1}+c_{2}\right) W_{4}^{2}\right]=E
$$

with the condition for Helmholtz separability,

$$
\partial_{12} \ln \left(K_{1}-K_{2}\right)=0 \text {. }
$$

Here

$$
R_{12}=\frac{3}{2} \frac{K_{1}^{\prime} K_{2}^{\prime}}{\left(K_{1}-K_{2}\right)^{2}}+\frac{1}{2} b_{2}^{\prime \prime}-\frac{b_{2}^{\prime} K_{2}^{\prime}}{2\left(K_{1}-K_{2}\right)}
$$

so the condition $R_{12}=0$ implies (2.15) although (2.15) doesn't imply $R_{12}=0$.

The third possibility is

$$
\frac{1}{K_{1}-K_{2}}\left[2 W_{1} W_{4}+2 W_{2} W_{3}+c_{1} W_{3}^{2}+d_{2} W_{4}^{2}\right]=E
$$

with the condition for Helmholtz separability

$$
\partial_{12} \ln \left(K_{1}-K_{2}\right)=0
$$

which is equivalent to $R_{12}=0$.

\section{F. One Ignorable Variable with Three Essential Variables of Type 2}

The HJ equation is

$$
\begin{aligned}
{[\mathrm{F}] \quad } & \frac{1}{\dot{S}}\left[\left(q_{2}-q_{3}\right) W_{1}^{2}+\left(q_{3}-q_{1}\right) W_{2}^{2}+\left(q_{1}-q_{2}\right) W_{3}^{2}\right. \\
& \left.+\left[r_{1}\left(q_{2}-q_{3}\right)+r_{2}\left(q_{3}-q_{1}\right)+r_{3}\left(q_{1}-q_{2}\right)\right] W_{4}^{2}\right]=E, \\
& S=s_{1}\left(q_{2}-q_{3}\right)+s_{2}\left(q_{3}-q_{1}\right)+s_{3}\left(q_{1}-q_{2}\right) .
\end{aligned}
$$


Since these coordinates are orthogonal the conditions for Helmholtz separability are $R_{i j}=0$ for all $i \neq j$. However, the condition $R_{l 4}=0, l=1,2,3$, is satisfied automatically.

G. One Ignorable Variable with One Essential Coordinate of Type 1 and Two Coordinates of Type 2

There are two cases to consider, the first being

$$
\begin{aligned}
{[\mathrm{G} 1] } & \frac{1}{Q}\left[W_{1}^{2}+W_{2}^{2}+2\left(l_{1}-l_{2}\right) W_{3} W_{4}+\left(m_{1}-m_{2}\right) W_{4}^{2}\right]=E, \\
& Q=k_{1}-k_{2}+g_{3}\left(l_{1}-l_{2}\right) .
\end{aligned}
$$

The conditions for Helmholtz separability are equivalent to

$$
\partial_{i j} \ln \left(\frac{Q}{l_{1}-l_{2}}\right)=0, \quad 1 \leqq i<j \leqq 3
$$

which are precisely $R_{i j}=0$.

The second case is

$$
\begin{aligned}
& \frac{1}{Q}\left[g_{3} W_{1}^{2}+l_{3} W_{2}^{2}+2 W_{3} W_{4}+\left(u_{2} l_{3}+g_{3} f_{1}\right) W_{4}^{2}\right]=E, \\
& Q=k_{3}+v_{2} l_{3}+g_{3} r_{1} .
\end{aligned}
$$

The conditions for Helmholtz separability are

$$
\partial_{i j} \ln Q=0, \quad 1 \leqq i<j \leqq 3
$$

which are precisely $R_{i j}=0$.

\section{H. No Ignorable Variables}

This is the most complicated case and the metric is necessarily orthogonal. The HJ Equation reads

$$
\text { [H] } \quad \sum_{j=1}^{4} \frac{M_{j 1}}{S} W_{j}^{2}=E
$$

where $S$ is a Stäckel determinant and $M_{j 1}$ is the (j1) cofactor of $S$. Eisenhart [11] has shown quite generally that the corresponding Helmholtz equation separates if and only if $R_{i j}=0$ for all $i \neq j$. A detailed classification of these coordinates will be given in Section 4.

Theorem 1. A separable coordinate system for the $\mathrm{HJ}$ equation in a four dimensional Riemannian space is equivalent to exactly one of the systems $\left\{x^{l}\right\}$ of types $\mathrm{A}-\mathrm{H}$. If $\left\{x^{l}\right\}$ is not of type $\mathrm{E} 1$ and $R_{i j}=0$ for all pairs of distinct essential variables $x^{i}, x^{j}$, then $\left\{x^{l}\right\}$ also separates the corresponding Helmholtz equation. Except for the coordinates of types $\mathrm{E} 1$ and $\mathrm{E} 2$ this condition is also necessary for Helmholtz separation. 
Since the Ricci tensor vanishes in flat space the following result is an immediate consequence of Theorem 1 and Lemma 2.

Corollary 1. A coordinate system in four dimensional flat space provides a separation of variables for the $\mathrm{HJ}$ equation

$$
\sum_{j=1}^{4}\left(\frac{\partial W}{\partial z^{j}}\right)^{2}=E
$$

if and only if it provides separation for the Helmholtz equation

$$
\sum_{j=1}^{4} \frac{\partial^{2} \psi}{\partial\left(z^{j}\right)^{2}}=E \psi
$$

This result is well known for orthogonal separable systems [11], but we have extended it to nonorthogonal systems.

Defining the Einstein tensor $G_{i j}=R_{i j}-\frac{1}{2} R g_{i j}$ it follows immediately from Theorem 1 that

Corollary 2. Let $\left\{x^{l}\right\}$ be a system, not of type E1, which separates variables in the HJ Equation and such that the vacuum Einstein equations $G_{i j}=0$ are satisfied. Then $\left\{x^{l}\right\}$ separates variables in the Helmholtz equation.

\section{Equations with Partial Separation}

We next enumerate the systems $\left\{x^{l}\right\}$ for the HJ and Helmholtz Equations such that one or two variables can be separated from the rest but the variables are not totally separable. We should emphasize that while these systems can be considered as generalizations of the coordinates of types A-G listed in Section 2, they are not true generalizations of type $\mathrm{H}$ coordinates. Indeed the most interesting and complicated of the type $\mathrm{H}$ coordinates (such as ellipsoidal coordinates) do not permit the splitting of one or two variables from the remaining variables. For these coordinates all variables must be separated simultaneously.

The seven types of partially separable systems will now be classified in terms of essential and ignorable variables.

\section{One Essential Variable of Type 2}

The HJ equation can be written as

$$
\frac{1}{K_{1}\left(x^{1}\right)+K\left(x^{2}, x^{3}, x^{4}\right)}\left[W_{1}^{2}+\sum_{j, l=2}^{4} A_{j l}\left(x^{2}, x^{3}, x^{4}\right) W_{j} W_{l}\right]=E .
$$

The condition for partial Helmholtz separation is that either $K_{1}$ or $K$ is constant, which is equivalent to $R_{1 j}=0, j=2,3,4$.

\section{J. One Ignorable Variable}

The HJ equation has the form

$$
[\mathrm{J}] \quad \sum_{j, l=1}^{4} G^{j l} W_{j} W_{l}=E
$$


where $\partial G^{j l} / \partial x^{1}=0$. The corresponding Helmholtz equation also admits partial separation in $x^{1}$.

K. Two Essential Variables of Type 2

The HJ equation has the form

$$
\begin{aligned}
& \frac{1}{Q}\left[G\left(x^{3}, x^{4}\right) W_{1}^{2}+H\left(x^{3}, x^{4}\right) W_{2}^{2}+\sum_{j, l=3}^{4} A_{j l}\left(x^{3}, x^{4}\right) W_{j} W_{l}\right]=E \\
& Q=K_{1}\left(x^{1}\right) G+K_{2}\left(x^{2}\right) H+K\left(x^{3}, x^{4}\right)
\end{aligned}
$$

and the condition for partial Helmholtz separability is that two of the functions $K_{1}, K_{2}, K$ are constant, which is equivalent to $R_{12}=0, R_{i j}=0, i=1,2, j=3,4$.

\section{Two Ignorable Variables}

The HJ equation is

$$
\sum_{j, l=1}^{4} G^{j l}\left(x^{3}, x^{4}\right) W_{j} W_{l}=E
$$

and the corresponding Helmholtz equation is also partially separable.

\section{One Essential Variable of Type 2 and One Ignorable Variable}

The $\mathrm{HJ}$ equation has the form

$$
[\mathrm{M}] \quad \frac{1}{K_{1}\left(x^{1}\right)+K\left(x^{3}, x^{4}\right)}\left[W_{1}^{2}+\sum_{j, l=2}^{4} A_{j l}\left(x^{3}, x^{4}\right) W_{j} W_{l}\right]=E
$$

and partial Helmholtz separability is achieved if either $K_{1}$ or $K$ is constant, which is equivalent to $R_{1 j}=0, j=3,4$.

\section{N. One Essential Variable of Type 1 and One Ignorable Variable}

The $\mathrm{HJ}$ equation has the form

$$
[\mathrm{N}] \quad \frac{1}{K_{1}\left(x^{1}\right)+K\left(x^{3}, x^{4}\right)}\left[2 W_{1} W_{2}+\sum_{j, l=2}^{4} A_{j l}\left(x^{3}, x^{4}\right) W_{j} W_{l}\right]=E
$$

and partial Helmholtz separability is achieved if either $K_{1}$ or $K$ is constant. In this case Helmholtz separability appears unrelated to any Ricci tensor condition.

O. Variable Splitting

Here the $\mathrm{HJ}$ equation becomes

$$
\begin{gathered}
\text { [O] } \frac{1}{K\left(x^{1}, x^{2}\right)+L\left(x^{3}, x^{4}\right)}\left[\sum_{j, l=1}^{2} A_{j l}\left(x^{1}, x^{2}\right) W_{j} W_{l}+\sum_{i, h=3}^{4} B_{i h}\left(x^{3}, x^{4}\right)\right. \\
\left.W_{i} W_{h}\right]=E
\end{gathered}
$$


and the corresponding Helmholtz equation partially separates if either $K$ or $L$ is constant, which is equivalent to $R_{i j}=0, i=1,2, j=3,4$.

\section{Equations with No Ignorable Variables}

We now present a detailed classification of the Helmholtz separable systems of type $H$. These are orthogonal systems $\left\{x^{m}\right\}$ for which the metric can be written

$$
d s^{2}=\sum_{j=1}^{4} H_{j}^{2}\left(d x^{j}\right)^{2}, H_{j}=S / M_{j 1},
$$

$S$ is a Stäckel determinant and $M_{j 1}$ is the $(j, 1)$ cofactor of $S$. Furthermore, none of the variables $x^{l}$ is ignorable and the Robertson condition $R_{j k}=0, j \neq k$ is satisfied. As is well-known [11] the Stäckel form condition (4.1) is equivalent to the system of equations

$$
\begin{gathered}
\partial_{j k} \ln H_{i}^{2}-\partial_{j} \ln H_{i}^{2}+\partial_{j} \ln H_{i}^{2} \partial_{k} \ln H_{j}^{2} \\
\quad+\partial_{k} \ln H_{i}^{2} \partial_{j} \ln H_{k}^{2}=0, \quad(j \neq k),
\end{gathered}
$$

and the Robertson condition reads

$$
R_{j k}=\frac{3}{4} \partial_{j k} \ln \left(H_{i}^{2} H_{l}^{2}\right)=0, \quad(j, k, i, l \neq) .
$$

From (4.2) we have $\partial_{j k} \ln \left(H_{j}^{2} / H_{k}^{2}\right)=0$. Combining this result with (4.3) we find

$$
\begin{aligned}
& H_{j}^{2}=\varphi_{j k} \Theta_{j k}, H_{k}^{2}=\varphi_{k j} \Theta_{j k} \\
& \varphi_{j k} \varphi_{k j} \Theta_{j k}^{2}=\psi_{i j k} \psi_{l j k},(j, k, i, l \neq) \\
& \partial_{k} \varphi_{j k}=\partial_{j} \varphi_{k j}=\partial_{l} \psi_{i j k}=\partial_{i} \psi_{l j k}=0, \\
& \Theta_{j k}=\Theta_{k j}, \psi_{i j k}=\psi_{i k j} .
\end{aligned}
$$

Furthermore,

$$
\partial_{l k i} \ln H_{l}^{2}=\partial_{l k i} \ln H_{k}^{2}=\partial_{l k i} \ln H_{i}^{2}=-\partial_{l k i} \ln H_{j}^{2}=f_{j}
$$

where $\partial_{j} f_{j}=0$. Integrating (4.5) and making use of (4.4) we see that $\ln H_{s}^{2}$ is a sum of functions, each function depending on at most two of the four variables $x^{i}, x^{j}, x^{k}, x^{l}$. Substituting this form for $\ln H_{m}^{2}$ into (4.4) we obtain

Lemma 3. If the metric $d s^{2}$ is in Stäckel form and satisfies the Robertson condition, then there exist nonzero functions $X_{l}=X_{l}\left(x^{l}\right), \xi_{i j}=\xi_{i j}\left(x^{i}, x^{j}\right)=\xi_{i i}, \eta_{i j}=\eta_{i j}\left(x^{i}, x^{j}\right)=$ $\eta_{j i}$ such that

$$
\begin{aligned}
& H_{1}^{2}=X_{1} \xi_{34} \xi_{23} \xi_{24} \eta_{31} \eta_{41} \eta_{21} \\
& H_{2}^{2}=X_{2} \xi_{34}^{-1} \xi_{13} \xi_{14} \eta_{42} \eta_{32} \eta_{21} \\
& H_{3}^{2}=X_{3} \xi_{12} \xi_{14}^{-1} \xi_{24}^{-1} \eta_{31} \eta_{32} \eta_{34} \\
& H_{4}^{2}=X_{4} \xi_{12}^{-1} \xi_{13}^{-1} \eta_{41} \eta_{42} \eta_{34} .
\end{aligned}
$$


Here

$$
\partial_{j k}\left(\xi_{j i} \xi_{j l} \xi_{k i} \xi_{k l} \eta_{j k}\right)=0, \quad(j, k, i, l \neq)
$$

Property (4.7) follows from substitution of (4.6) into (4.2) for $i=j$. The nonzero functions $X_{i}$ are arbitrary and can be modified at will by the trivial change of variable $x^{m^{\prime}}=x^{m^{\prime}}\left(x^{m}\right), m=1, \ldots, 4$, which does not affect variable separation.

To complete our classification we need only satisfy the Equations (4.2) for $i, j, k$ distinct. Note that the $\xi_{i j}$ and $\eta_{i j}$ are not uniquely determined by (4.6). Indeed these expressions are invariant under the replacements

$$
\begin{array}{ll}
\xi_{12} \rightarrow a_{1} c_{2} \xi_{12} & \eta_{21} \rightarrow a_{2} b_{2} b_{1} c_{1} \eta_{21} \\
\xi_{13} \rightarrow b_{1} c_{3} \xi_{13} & \eta_{31} \rightarrow a_{3} b_{3} a_{1} c_{1}^{-1} \eta_{31} \\
\xi_{14} \rightarrow c_{1} c_{4} \xi_{14} & \eta_{41} \rightarrow a_{4} b_{4} a_{1}^{-1} b_{1}^{-1} \eta_{41} \\
\xi_{23} \rightarrow a_{2} b_{3} \xi_{23} & \eta_{32} \rightarrow a_{3}^{-1} c_{3} c_{2} b_{2}^{-1} \eta_{32} \\
\xi_{24} \rightarrow b_{2} b_{4} \xi_{24} & \eta_{42} \rightarrow a_{4}^{-1} c_{4} a_{2}^{-1} c_{2}^{-1} \eta_{42} \\
\xi_{34} \rightarrow a_{3} a_{4} \xi_{34} & \eta_{43} \rightarrow c_{4}^{-1} b_{4}^{-1} b_{3}^{-1} c_{3}^{-1} \eta_{43}
\end{array}
$$

where $a_{i}, b_{i}, c_{i}$ are nonzero functions of the single variable $x^{i}$. In particular, if $\partial_{j k} \ln \xi_{j k}=0$ for some $j, k$ then without loss of generality we can assume $\xi_{j k}=1$.

Denote each of the twelve Equations (4.2) for $i, j, k$ distinct by $[i ; j, k](\equiv[i ; k, j])$. Differentiating each equation $[i ; j, k]$ with respect to $x^{i}$ and $x^{l}$ where $j, k, i, l$ are distinct, we obtain twelve equations which are equivalent to the eight conditions

$$
\begin{aligned}
& A_{12}\left(A_{34}+2 B_{34}\right)+A_{13}\left(A_{24}+2 B_{24}\right)=0 \\
& A_{34}\left(A_{12}-2 B_{12}\right)+A_{24}\left(A_{13}-2 B_{13}\right)=0 \\
& A_{23}\left(A_{14}-2 B_{14}\right)-A_{34}\left(A_{12}+2 B_{12}\right)=0 \\
& A_{12}\left(A_{34}+2 B_{34}\right)-A_{14}\left(A_{23}+2 B_{23}\right)=0 \\
& A_{14}\left(A_{23}-2 B_{23}\right)+A_{24}\left(A_{13}+2 B_{13}\right)=0 \\
& A_{23}\left(A_{14}-2 B_{14}\right)+A_{13}\left(A_{24}-2 B_{24}\right)=0 \\
& A_{12}\left(A_{34}-2 B_{34}\right)-A_{23}\left(A_{14}+2 B_{14}\right)=0 \\
& A_{12}\left(A_{34}-2 B_{34}\right)+A_{24}\left(A_{13}+2 B_{13}\right)=0 \\
& A_{i j}=\partial_{i j} \ln \xi_{i j}, B_{i j}=\partial_{i j} \ln \eta_{i j} .
\end{aligned}
$$

Our analysis of the possible Helmholtz separable systems depends strongly on which of the various factors vanish in expressions (4.9). We examine all possible cases.

Case 1. $A_{i j} \pm 2 B_{i j} \equiv 0$ for all $i, j$.

Then $A_{i j} \equiv 0$ and we can assume that $\xi_{i j} \equiv 1$.

Case 2. $A_{i j}\left(A_{k l} \pm 2 B_{k l}\right) \neq 0$ for some choice of $i, j, k, l$.

To be definite we assume $(i, j, k, l)=(1,3,2,4)$. Then it follows from (4.9) that 
$A_{12}, A_{23}, A_{34}$ and $A_{14}$ are nonzero, as well as $A_{34}+2 B_{34}, A_{14}-2 B_{14}, A_{12}+$ $2 B_{12}, A_{23}+2 B_{23}$. Furthermore, relations (4.9) imply that $\partial_{12} \ln A_{12}=\partial_{23} \ln$ $A_{23}=\partial_{34} \ln A_{34}=\partial_{14} \ln A_{14}=0$. Integrating these equations and substituting into

$$
\partial_{12}\left(\xi_{13} \xi_{14} \xi_{23} \xi_{24} \eta_{12}\right)=0,
$$

a special case of (4.7), we see that this last condition cannot be satisfied. Thus Case 2 doesn't occur.

Case 3. Case 2 doesn't hold but for some choice of distinct $i, j, k, l, A_{i j}\left(A_{k l}+2 B_{k l}\right) \neq$ $0, A_{k l}-2 B_{k l}=0$.

Without loss of generality we can assume $(i, j, k, l)=(1,3,2,4)$. It follows from (4.9) that $A_{12}, A_{34}+2 B_{34}, A_{14}, A_{23}+2 B_{23}$ are nonzero. Since Case 2 doesn't hold, we have $A_{34}=2 B_{34} \neq 0, A_{23}=2 B_{23} \neq 0, A_{14}=-2 B_{14}$ and $A_{13} \pm 2 B_{13}=0$. This last pair of equalities implies $A_{13}=0$, which is impossible.

Case 4. Case 2 doesn't hold but for some choice of distinct $i, j, k, l, A_{i j}\left(A_{k l}-\right.$ $\left.2 B_{k l}\right) \neq 0, A_{k l}+2 B_{k l}=0$.

By a computation analogous to Case 3 we can show that this is impossible.

It follows from the above that

$$
A_{i j}\left(A_{k l} \pm 2 B_{k l}\right)=0
$$

for all distinct $i, j, k, l$. The possibility that the second factor is always zero has been treated in Case 1. We now treat the remaining possibilities.

Case 5. $A_{i j} \equiv 0$ for all distinct $i, j$.

Here we can assume that $\xi_{i j} \equiv 1$.

Case 6. $A_{i j}, A_{i k}, A_{i l} \neq 0, i, j, k, l$ distinct.

Without loss of generality we can assume that $(i, j, k, l)=(1,2,3,4)$. Then $A_{12}, A_{13}, A_{14} \neq 0$ and from (4.10) $A_{34}=A_{24}=A_{23}=B_{34}=B_{24}=B_{23}=0$. We can use (4.8) and assume without loss of generality that $\eta_{34}=\eta_{24}=\eta_{23}=1$, $\xi_{34}=1, \xi_{24}=\xi_{24}\left(x^{4}\right)$. Differentiating equation $[3 ; 2,1]$, (4.2), with respect to $x^{4}$ and $[4 ; 2,1]$ with respect to $x^{3}$ we obtain

$$
\begin{aligned}
& A_{14}\left(2 \partial_{2} \ln \xi_{12}-\partial_{2} \ln \xi_{23}-\partial_{2} \ln \eta_{12}\right)=0 \\
& A_{13}\left(-2 \partial_{2} \ln \xi_{12}-3 \partial_{2} \ln \xi_{23}-\partial_{2} \ln \eta_{12}\right)=0
\end{aligned}
$$

which implies $A_{12}=B_{12}=0$, a contradiction.

Case 7. $A_{i j}, A_{j k}, A_{k i} \neq 0$.

Without loss of generality we can assume that $(i, j, k)=(1,2,4)$. Then $A_{12}, A_{24}$, $A_{41} \neq 0$ and $A_{34}=A_{13}=A_{23}=B_{13}=B_{34}=B_{23}=0$. We can use (4.8) and assume without loss of generality that $\eta_{34}=\eta_{31}=1, \xi_{34}=\xi_{34}\left(x^{3}\right), \xi_{31}=\xi_{31}\left(x^{3}\right)$, $\xi_{32}=\xi_{32}\left(x^{3}\right), \eta_{32}=\eta_{32}\left(x^{3}\right)$. Writing the simultaneous equations $\partial_{1}[4 ; 2,3]$, $\partial_{4}[2 ; 1,3], \partial_{2}[4 ; 1,3], \partial_{4}[1 ; 2,3], \partial_{2}[1 ; 3,4], \partial_{1}[2 ; 3,4]$ we see that these equations are consistent only if $\xi_{34}, \xi_{31}, \xi_{32}$ and $\eta_{32}$ are constants. Thus, $x^{3}$ is an ignorable variable, which is impossible. 
Case 8. $A_{i j}, A_{j k} \neq 0$, all other $A_{s t}=0$.

Without loss of generality we can assume $(i, j, k)=(1,2,4)$. Then $A_{12}, A_{24}$ are the only nonzero $A_{s t}$ and $B_{34}=B_{13}=0$. Employing (4.8) we can require $\eta_{34}=\eta_{13}=\xi_{34}=\xi_{14}=1, \partial_{1} \xi_{13}=\partial_{2} \xi_{23}=0$. Then equations $\partial_{1}[4 ; 2,3], \partial_{2}[4 ; 1$, $3], \partial_{4}[1 ; 2,3]$ are consistent only if $\partial_{3} \xi_{13}=\partial_{3} \xi_{23}=\partial_{3} \eta_{23}=0$. It follows that $x^{3}$ is ignorable and this is a contradiction.

Case 9. Only one nonzero function $A_{i j}$.

Without loss of generality we can assume that $A_{12}$ is the only nonzero $A$. Thus $B_{34}=0$ and by employing (4.8) we can require $\eta_{34}=\xi_{34}=\xi_{14}=1, \partial_{2} \xi_{24}=0$. From equations $\partial_{1}[3 ; 2,4]$ and $\partial_{2}[3 ; 1,4]$ we find that $\partial_{4} \ln \eta_{24}=-2 \partial_{4} \ln \xi_{24}$, $\partial_{4} \ln \eta_{14}=-3 \partial_{4} \ln \xi_{24}, B_{14}=B_{24}=0$ and by (4.8) we can require $\partial_{1} \eta_{14}=$ $\partial_{2} \eta_{24}=0$. The additional condition $\partial_{34}\left(\xi_{13} \xi_{23} \xi_{14} \xi_{24} \eta_{34}\right)=0$ from (4.7) implies $\partial_{4} \xi_{24}=0$ so $\xi_{24}, \eta_{14}$ and $\eta_{24}$ are constants. It follows that $x^{4}$ is ignorable, which is a contradiction.

We have strengthened Lemma 3 to

Lemma 4. If the metric $d s^{2}$ satisfies the hypotheses of Lemma 3 and admits no ignorable coordinates, then

$$
\begin{aligned}
& H_{i}^{2}=X_{i}\left(x^{i}\right) \prod_{j \neq i} \eta_{j i}\left(x^{j}, x^{i}\right), \eta_{j i}=\eta_{i j}, 1 \leqq i \leqq 4 \\
& \partial_{k l} \eta_{k l}=0,1 \leqq k<l \leqq 4
\end{aligned}
$$

In consequence of the Stäckel conditions (4.2), the components of the Riemann curvature tensor for $i, j, k$ distinct may be written [11]

$$
R_{j i i k}=\frac{3}{4} H_{i}^{2} \partial_{j k} \ln H_{i}^{2} \text {. }
$$

An immediate consequence of Lemma 4 is

Lemma 5. If $d s^{2}$ satisfies the hypotheses of Lemma 3 and admits no ignorable coordinates, then $R_{j i i k}=0$.

It is remarkable that the strong condition $R_{j i i k}=0$ is automatically satisfied by a Helmholtz separable system with no ignorable variables. In Ref. [18] two of the authors computed all Helmholtz separable systems in four variables such that the technical condition $R_{j i i k}=0$ was satisfied. The results were also reported in Refs. [1], [17] and were obtained by substituting expressions (4.11) into the twelve equations $[i ; j, k]$ and solving for the $\eta_{i j}$. We now see that the answer to our present problem can be obtained by eliminating the separable metrics with ignorable variables from our previous list. Thus we have

Theorem 2. The metric $d s^{2}=\sum_{1}^{4} H_{i}^{2}\left(d x^{i}\right)^{2}$ defines a Helmholtz separable system with no ignorable variables if and only if the metric coefficients take one of the following forms:

$$
\begin{array}{ll}
{[\mathrm{Ha}] \quad H_{1}^{2}} & =X_{1}\left(\sigma_{1}-\sigma_{2}\right), H_{2}^{2}=X_{2}\left(\sigma_{1}-\sigma_{2}\right) \\
H_{3}^{2} & =X_{3}\left(\sigma_{3}-\sigma_{4}\right), H_{4}^{2}=X_{4}\left(\sigma_{3}-\sigma_{4}\right) \\
\sigma_{i} & =\sigma_{i}\left(x^{i}\right), \sigma_{i}^{\prime} \neq 0,
\end{array}
$$


[Hb] $\quad H_{1}^{2}=X_{1}\left(\sigma_{1}-\sigma_{2}\right), H_{2}^{2}=X_{2}\left(\sigma_{1}-\sigma_{2}\right)$

$$
\begin{aligned}
H_{3}^{2} & =X_{3} \sigma_{1} \sigma_{2}\left(\sigma_{3}-\sigma_{4}\right), H_{4}^{2}=X_{4} \sigma_{1} \sigma_{2}\left(\sigma_{3}-\sigma_{4}\right) \\
\sigma_{i}^{\prime} & \neq 0,
\end{aligned}
$$

[Hc] $\quad H_{i}^{2}=X_{i}\left(\sigma_{i}-\sigma_{j}\right)\left(\sigma_{i}-\sigma_{k}\right)\left(\sigma_{i}-\sigma_{l}\right)$

with $i, j, k$, ldistinct,

[Hd]

$$
\begin{aligned}
& H_{1}^{2}=X_{1}, H_{2}^{2}=X_{2} \varphi_{1}\left(\sigma_{23}+\sigma_{32}\right)\left(\sigma_{24}+\sigma_{42}\right) \\
& H_{3}^{2}=X_{3} \varphi_{1}\left(\sigma_{32}+\sigma_{23}\right)\left(\sigma_{34}+\sigma_{43}\right), H_{4}^{2}=X_{4} \varphi_{1}\left(\sigma_{42}+\sigma_{24}\right)\left(\sigma_{43}+\sigma_{34}\right), \\
& \varphi_{1}^{\prime} \neq 0 .
\end{aligned}
$$

Here $\sigma_{i j}$ is a function of $x^{i}$ alone.

We will now use our results to prove a theorem about Ricci-flat spaces, i.e. complex Riemannian spaces which satisfy the vacuum Einstein equations. (At this point it is convenient to write $x_{i}$ in place of $x^{i}$.)

Theorem 3. If in a Ricci-flat $\left(R_{i j}=0\right)$ complex Riemannian space the $\mathrm{HJ}$ equation admits a separation of variables with no ignorable coordinates, then the space is flat.

Before proving this we prove a simple but useful lemma.

Lemma 6. Suppose the metric $d s^{2}$ is in Stäckel form, is Ricci flat (i.e. $R_{i j}=0$ ), and admits no ignorable coordinates. Furthermore, suppose that for a fixed value of $i$ $R_{i j j i}=0$ for two values of $j \neq i$. Then the space is flat.

Proof. Since $R_{i j}=0$ implies the Robertson condition, it follows from Lemma 5 that $R_{j i i k}=0$. Now without loss of generality we take as our two vanishing components of $R_{i j j i}, R_{1221}=R_{1331}=0$. It follows immediately from $R_{11}=0$ that $R_{1441}=0$. The remaining Ricci equations are

$$
\begin{aligned}
& R_{22}=\frac{R_{2332}}{H_{3}^{2}}+\frac{R_{2442}}{H_{4}^{2}}=0 \\
& R_{33}=\frac{R_{2332}}{H_{2}^{2}}+\frac{R_{3443}}{H_{4}^{2}}=0 \\
& R_{44}=\frac{R_{2442}}{H_{2}^{2}}+\frac{R_{3443}}{H_{3}^{2}}=0 .
\end{aligned}
$$

It is easy to see that the only solution to these equations is $R_{2332}=R_{2442}=$ $R_{3443}=0$. Q.E.D. p. 44).

In order to prove Theorem 3 we will need the following expression ([19],

$$
\begin{aligned}
R_{j i i j}= & H_{i}^{2}\left(\partial_{j j} \ln H_{i}+\partial_{j} \ln H_{i} \partial_{j} \ln \frac{H_{i}}{H_{j}}\right) \\
& +H_{j}^{2}\left(\partial_{i i} \ln H_{j}+\partial_{i} \ln H_{j} \partial_{i} \ln \frac{H_{j}}{H_{i}}\right) \\
& +\sum_{h \neq j, i} \frac{H_{i}^{2} H_{j}^{2}}{H_{k}^{2}} \partial_{k} \ln H_{i} \partial_{k} \ln H_{j} .
\end{aligned}
$$


Proof of Theorem 3. Since $R_{i j}=0 \mathrm{HJ}$ separation implies Helmholtz separation and by Theorem 2 we need only prove the result for the four types of metrics [Ha-d]. We proceed by cases:

(Ha) It is easy to see from (4.13) that $R_{1331}=R_{1441}=0$ and by Lemma $6 R_{i j k l}=0$. (Hb) If either $\sigma_{1}$ or $\sigma_{2}$ is constant, we can redefine variables to obtain an ignorable coordinate. Thus, $\sigma_{1}, \sigma_{2}$ are not constant and we redefine $x_{1}, x_{2}$ such that $H_{1}^{2}=$ $X_{1}\left(x_{1}-x_{2}\right), H_{2}^{2}=X_{2}\left(x_{1}-x_{2}\right), H_{3}^{2}=X_{3} x_{1} x_{2}\left(\sigma_{3}-\sigma_{4}\right), H_{4}^{2}=X_{4} x_{1} x_{2}\left(\sigma_{3}-\sigma_{4}\right)$. From (4.13) we find

$$
\frac{R_{1221}}{H_{2}^{2}}=\frac{1}{2\left(x_{1}-x_{2}\right)}\left[-\frac{1}{x_{1}-x_{2}}\left(\frac{X_{1}}{X_{2}}+1\right)+\frac{1}{2}\left(\left(\frac{1}{X_{1}}\right)^{\prime}-\left(\frac{1}{X_{2}}\right)^{\prime}\right) X_{1}\right]
$$

and

$$
\frac{R_{1331}}{H_{3}^{2}}=\frac{R_{1441}}{H_{4}^{2}}=-\frac{1}{4}\left[\frac{1}{x_{1}^{2}}+\frac{1}{x_{1}\left(x_{1}-x_{2}\right)}+\frac{1}{x_{1}} \frac{X_{1}^{\prime}}{X_{1}}+\frac{1}{x_{2}\left(x_{1}-x_{2}\right)} \frac{X_{1}}{X_{2}}\right] .
$$

Forming the Ricci component

$$
R_{11}=\frac{R_{1221}}{H_{2}^{2}}+\frac{R_{1331}}{H_{3}^{2}}+\frac{R_{1441}}{H_{4}^{2}}
$$

we find

$$
\begin{aligned}
2 R_{11}=- & \frac{1}{\left(x_{1}-x_{2}\right)^{2}}-\frac{1}{x_{1}\left(x_{1}-x_{2}\right)}-\frac{1}{x_{1}^{2}}-\frac{x_{1}}{x_{2}\left(x_{1}-x_{2}\right)} \frac{X_{1}}{X_{2}}-\frac{1}{x_{1}} \frac{X_{1}^{\prime}}{X_{1}} \\
& +\frac{X_{1}}{2\left(x_{1}-x_{2}\right)}\left[\left(\frac{1}{X_{1}}\right)^{\prime}-\left(\frac{1}{X_{2}}\right)^{\prime}\right]=0 .
\end{aligned}
$$

Consider the operator $\left(x_{1}-x_{2}\right)^{2} \partial_{2}=A$. Computing $A^{3} R_{11}=0$ we obtain an expression independent of $X_{1}$. Equating, then, coefficients of powers of $x_{1}$ to zero, we find $\left(\frac{1}{X_{2}}\right)^{(3)}=0$. By symmetry of the coordinates $x_{1}$ and $x_{2}$, we conclude $\left(\frac{1}{X_{1}}\right)^{(3)}=0$. Plugging this information back into $R_{11}=0$, we find

$$
\frac{1}{X_{1}}=c x_{1}^{2}+d x_{1}, \quad \frac{1}{X_{2}}=-\left(c x_{2}^{2}+d x_{2}\right)
$$

from which $R_{1331}=R_{1441}=0$. Thus the desired result follows by Lemma 6 . $(\mathrm{Hc})$ This is the most difficult case. We can assume that no $\sigma_{i}$ is constant; for if a $\sigma_{i}$ were constant, we could find an ignorable coordinate. Thus we can take $\sigma_{i}=x_{i}$, and from (4.13) we find

$$
\begin{aligned}
\frac{2 R_{i j j i}}{H_{i}^{2} H_{j}^{2}} & =-\frac{1}{\left(x_{i}-x_{j}\right)\left(x_{i}-x_{k}\right)\left(x_{i}-x_{l}\right)}\left(\frac{-1}{\left(x_{i}-x_{j}\right)^{2}}+\frac{1}{2\left(x_{i}-x_{j}\right)\left(x_{i}-x_{k}\right)}\right. \\
& \left.+\frac{1}{2\left(x_{i}-x_{j}\right)\left(x_{i}-x_{l}\right)}\right)\left(\frac{1}{X_{i}}\right)+\frac{1}{2\left(x_{i}-x_{j}\right)^{2}\left(x_{i}-x_{k}\right)\left(x_{i}-x_{l}\right)}\left(\frac{1}{X_{i}}\right)^{\prime}+
\end{aligned}
$$




$$
\begin{aligned}
& +\frac{1}{2\left(x_{i}-x_{j}\right)^{2}\left(x_{j}-x_{k}\right)\left(x_{j}-x_{l}\right)}\left(\frac{1}{X_{j}}\right)^{\prime} \\
& +\frac{1}{\left(x_{j}-x_{i}\right)\left(x_{j}-x_{k}\right)\left(x_{j}-x_{l}\right)}\left(-\frac{1}{\left(x_{i}-x_{j}\right)^{2}}+\frac{1}{2\left(x_{i}-x_{j}\right)\left(x_{j}-x_{k}\right)}\right. \\
& \left.\left.+\frac{1}{2\left(x_{i}-x_{j}\right)\left(x_{j}-x_{l}\right.}\right)\left(\frac{1}{X_{j}}\right)+\frac{1}{2\left(x_{k}-x_{i}\right)^{2}\left(x_{k}-x_{j}\right)^{2}\left(x_{k}-x_{l}\right)}\right)\left(\frac{1}{X_{k}}\right) \\
& +\frac{1}{2\left(x_{l}-x_{i}\right)^{2}\left(x_{l}-x_{j}\right)^{2}\left(x_{l}-x_{k}\right)}\left(\frac{1}{X_{l}}\right)
\end{aligned}
$$

for $i, j, k, l$ different. Computing the Ricci component $R_{11}$ from (4.14) we find

$$
\begin{aligned}
\frac{2 R_{11}}{H_{1}^{2}}= & \frac{1}{\left(x_{1}-x_{2}\right)\left(x_{1}-x_{3}\right)\left(x_{1}-x_{4}\right)}\left\{-\left(\frac{1}{X_{1}}\right) \sum\left(\frac{1}{\left(x_{1}-x_{j}\right)^{2}}+\frac{1}{\left(x_{1}-x_{j}\right)\left(x_{1}-x_{k}\right)}\right)\right. \\
& \left.+\frac{1}{2}\left(\frac{1}{X_{1}}\right)^{\prime} \sum \frac{1}{x_{1}-x_{j}}\right\}+\sum \frac{\left[\frac{1}{2}\left(\frac{1}{X_{j}}\right)^{\prime}+\frac{1}{x_{1}-x_{j}}\left(\frac{1}{X_{j}}\right)\right]}{\left(x_{1}-x_{j}\right)^{2}\left(x_{j}-x_{k}\right)\left(x_{j}-x_{l}\right)}=0 .
\end{aligned}
$$

Multiplying this expression by $\left(x_{1}-x_{2}\right)^{3}\left(x_{2}-x_{3}\right)\left(x_{2}-x_{4}\right)$ and differentiating five times with respect to $x_{2}$, we obtain $\left(\frac{1}{X_{2}}\right)^{(5)}=0$. By symmetry we conclude

$$
\left(\frac{1}{X_{l}}\right)^{(5)}=0
$$

Plugging this information back into $R_{11}$, we see by equating powers that the coefficients in $\left(\frac{1}{X_{l}}\right)$ of the powers of $x_{i}$ are independent of $i$, i.e.

$$
\left(\frac{1}{X_{i}}\right)=a x_{i}^{4}+b x_{i}^{3}+c x_{i}^{2}+d x_{i}+e_{i} \text {. }
$$

A straightforward but tedious computation then shows that $R_{1 i i 1}=0$, and our result follows from Lemma 6.

(Hd) Notice that if $\varphi_{1}$ is constant we can make $x_{1}$ an ignorable coordinate. Assuming $\varphi_{1} \neq$ constant we redefine $x_{1}$ so that $\varphi_{1}=x_{1}$. An easy computation using (4.13) shows that

$$
R_{1 i i 1}=-\frac{H_{i}^{2}}{4}\left(\frac{1}{x_{1}^{2}}+\frac{1}{x_{1}} \frac{X_{1}^{\prime}}{X_{1}}\right) \text {. }
$$

It follows from (4.14) that

$$
R_{11}=-\frac{3}{4}\left(\frac{1}{x_{1}^{2}}+\frac{1}{x_{1}} \frac{X_{1}^{\prime}}{X_{1}}\right) \text {. }
$$

Thus $R_{11}=0$ implies $R_{1 i i 1}=0$ and invoking Lemma 6 once again we obtain the theorem.

We have the following simple result: 
Corollary 3. If in a Ricci-flat complex Riemannian space the Hamilton-Jacobi equation admits a separation of variables, then the space admits a Killing vector (i.e. an infinitesimal isometry).

\section{References}

1. Kalnins, E. G., Miller, W. Jr. : Lie theory and the wave equation in space time. IV. The KleinGordon equation and the Poincaré group. J. Math. Phys. (to appear)

2. Carter, B.: Hamilton-Jacobi and Schrödinger separable solutions of Einstein's equations. Commun. math. Phys. 10, 280-310 (1968)

3. Woodhouse, N. M. J. : Killing tensors and the separation of the Hamilton-Jacobi equations. Commun. math. Phys. 44, 9-38 (1975)

4. Dietz, W.: Separable coordinate systems for the Hamilton-Jacobi, Klein-Gordon and wave equations in curved spaces. J. Phys. A(GB) 9, 519-533 (1976)

5. Collinson, C. D., Fugère, J. : Empty space-times with separable Hamilton-Jacobi equation. J. Phys. A(GB) 10, 745-753 (1977)

6. Miller, W. Jr. : Symmetry and separation of variables. Reading, Massachusetts: Addison-Wesley 1977

7. Morse, P., Feshbach, H. : Methods of mathematical physics, Part 1. New York: McGraw-Hill 1953

8. Stäckel, P. : Habilitationsschrift, Halle (1891)

9. Kalnins, E. G., Miller, W. Jr. : Separable coordinates for three-dimensional complex Riemannian spaces. J. Diff. Geometry (to appear)

10. Kalnins, E. G., Miller, W. Jr. : The wave equation and separation of variables on the complex sphere $S_{4}$. (submitted)

11. Eisenhart, L. P. : Separable systems of Stäckel. Ann. Math. 35, 284-305 (1934)

12. Robertson, H. P. : Bemerkung über separierbare Systeme in der Wellenmechanik. Math. Ann. 98, 749-752 (1927)

13. Havas, P. : Separation of variables in the Hamilton-Jacobi, Schrödinger, and related equations. 1. Complete separation. J. Math. Phys. 16, 1461-1468 (1975)

14. Havas, P. : Separation of variables in the Hamilton-Jacobi, Schrödinger, and related equations. 2. Partial separation. J. Math. Phys. 16, 2476-2489 (1975)

15. Boyer, C. P., Kalnins, E. G., Miller, W. Jr. : Symmetry and separation of variables for the HamiltonJacobi equation $W_{t}^{2}-W_{x}^{2}-W_{y}^{2}=0$. J. Math. Phys. 19, 200-211 (1978)

16. Boyer, C. P., Kalnins, E. G., Miller, W. Jr. : R-separable coordinates for three-dimensional complex Riemannian spaces. T.A.M.S. (to appear)

17. Kalnins, E. G., Miller, W. Jr. : R-separation of variables for the four-dimensional flat space Laplace and Hamilton-Jacobi equations. T.A.M.S. (to appear)

18. Kalnins, E. G., Miller, W. Jr. : The Klein-Gordon equation and the Poincaré group. University of Waikato Research Report, No. 37 (1976)

19. Eisenhart, L. P. : Riemannian geometry. Princeton: Princeton University Press 1949 (2nd printing)

Communicated by J. Glimm

Received November 14, 1977 